\title{
Digital Phono- and Electro-Cardiography: Predicting Echocardiographic Parameters for Telemedicine Screening
}

\author{
S Khoor ${ }^{1}$, I Kovacs ${ }^{1}$, K Fugedi $^{1}$, Gy Horvath ${ }^{1}$, \\ E Domijan $^{2}, \mathrm{M}$ Domijan $^{2}$ \\ ${ }^{1}$ Szent Istvan Hospital, Budapest, Hungary \\ ${ }^{2}$ UVA Corporation, Toronto, Canada
}

\begin{abstract}
Our study was performed to assess the clinical value of digital phono- and electrocardiography (dPCG and $d E C G)$ in telemedicine application for screening. Relevant echocardiographic parameters (left ventricular $(L V)$, both atrial dimensions; ejection fraction $(E F)$, aortic Vmax, the grade of mitral and tricuspid regurgitation (MR and TR: semiquantitative grade I-III, the left ventricular mass index (LVMI)) were estimated from the $d P C G$ and $d E C G$ data. The study population consists of 790 patients selected from our database; 395 395 patients were in the training and the test groups. The signal analysis of the dPCG consists of: discrete FFT analysis; automated heart sound detection; filtering and averaging; multivariate discriminant analysis. The dPCG estimated Vmax, EF, MR and TR, the prediction based on dECG for LVIDd, LVMI, LA and RA dimensions showed highly significant results.
\end{abstract}

\section{Introduction}

Based on the comprehensive work of Tavel [1], our team decided to develop a large phono-echocardiographic and ECG database for the non-invasive estimation (modeling) of various heart disease and disease states [24]. The most important studies on clinical phonocardiography [5-7] did not use large database for the analysis, and it would be a serious limitation of their clinical usefulness. The advanced signal analysis, the potential telemedicine application may be give a new power of this old-new method.

\section{Methods}

The study population consists of 790 patients selected from our large database containing all of the relevant clinical parameters: the digital, 12-lead ECG, the 30second dPCG registration and the complete echocardiographic video. 395-395 patients were in the training and the test groups.
The automated measurement of the dECG and dPCG registrations ((TriTest device, sampling rate $1 \mathrm{kHz}$, in the range of $20-12000 \mathrm{kHz}$ ) were supervised by two cardiologists. The morphological parameters (amplitudes, time-intervals) of the ECG, and 170 phono-spectrogram measurements in one cardiac cycle were served for the further analysis.

\section{Results}

Both of the APCG and dECG analysis began with the factor analysis to reduce the large amount of input parameters. Table 1. shows the factor analysis of various ECG parameters in the case of sinus rhythm. Using the principal component analysis, 18 components were extracted. The F1 factor represents $13.58 \%$ of the variance, the cumulative percent of the first four's variance is $37.4 \%$. The most important parameters were used in the MDA.

Table 1. Factor analysis of ECG parameters (in sinus rhythm) (coefficients below 0.5 are zero-valued).

\begin{tabular}{|lrrrr|}
\hline \multicolumn{1}{|c}{ ECG } & F1 & \multicolumn{1}{c}{ F2 } & F3 & \multicolumn{1}{c|}{ F4 } \\
\hline & 1 & 2 & 3 & 4 \\
\hline LA_trans & 0 & -0.548 & 0 & 0 \\
\hline LA_Area & 0 & -0.611 & 0 & 0 \\
\hline RA_Iong & 0 & -0.626 & 0 & 0 \\
\hline RA_trans & 0 & -0.576 & 0 & 0 \\
\hline RA_Area & 0 & -0.66 & 0 & 0 \\
\hline R_I & 0 & 0 & -.551 & 0 \\
\hline R_II & 0 & 0.558 & 0 & 0 \\
\hline R_III & 0 & 0.535 & 0 & 0 \\
\hline R_aVFI & 0 & 0.588 & 0 & 0 \\
\hline R_V2 & 0 & 0 & 0 & 0.510 \\
\hline R_V4 & 0 & 0 & 0 & 0.574 \\
\hline R_V5 & 0 & 0 & 0 & 0.593 \\
\hline R_V6 & 0 & 0 & 0 & 0.532 \\
\hline S_II & 0 & 0 & 0 & 0.611 \\
\hline S_aVF & 0 & 0 & 0 & 0.547 \\
\hline S_V2 & 0 & 0 & 0.593 & 0 \\
\hline S_V3 & 0 & 0 & 0.664 & 0 \\
\hline S_V4 & 0 & 0 & 0.626 & 0 \\
\hline & & & &
\end{tabular}




\begin{tabular}{|lcrrr|}
\hline \multicolumn{1}{|c}{ ECG } & \multicolumn{1}{c}{ F1 } & F2 & F3 & F4 \\
\hline S_V5 & 0 & 0 & 0.518 & 0 \\
\hline $\begin{array}{l}\text { II_T-to- } \\
\text { P1minmax }\end{array}$ & 0.788 & 0 & 0 & 0 \\
\hline $\begin{array}{l}\text { II_P- } \\
\text { duration }\end{array}$ & 0.769 & 0 & 0 & 0 \\
\hline $\begin{array}{l}\text { III_T-to- } \\
\text { P1minmax }\end{array}$ & 0.756 & 0 & 0 & 0 \\
\hline $\begin{array}{l}\text { III_P- } \\
\text { duration }\end{array}$ & 0.766 & 0 & 0 & 0 \\
\hline $\begin{array}{l}\text { V1_T-to- } \\
\text { P1minmax }\end{array}$ & 0.639 & 0 & 0 & 0 \\
\hline $\begin{array}{l}\text { V1_P- } \\
\text { duration }\end{array}$ & 0.769 & 0 & 0 & 0 \\
\hline PII_1st_T & 0.788 & 0 & 0 & 0 \\
\hline PIII_1st_T & 0.756 & 0 & 0 & 0 \\
\hline PV1_1st_T & 0.639 & 0 & 0 & 0 \\
\hline
\end{tabular}

Table 2. Factor analysis of ECG parameters in atrial fibrillation (coefficients below 0.2 are zero-valued).

\begin{tabular}{|lrrrrrr|}
\hline & $\mathbf{1}$ & $\mathbf{2}$ & $\mathbf{3}$ & $\mathbf{4}$ & $\mathbf{5}$ & $\mathbf{6}$ \\
\hline QRS & 0 & 0 & 0.786 & 0 & 0 & 0 \\
\hline fII & 0 & 0 & 0 & 0.874 & 0 & 0 \\
\hline fIII & 0 & 0 & 0 & 0.957 & 0 & 0 \\
\hline faVF & 0 & 0 & 0 & 0.870 & 0 & 0 \\
\hline fV1 & 0 & 0 & 0 & 0 & 0.383 & 0 \\
\hline fV2 & 0 & 0 & 0 & 0 & 0.438 & 0 \\
\hline RII & 0 & 0.883 & 0 & 0 & 0 & 0 \\
\hline RIII & 0 & 0.805 & 0 & 0 & 0.276 & 0 \\
\hline RaVF & 0 & 0.846 & 0 & 0 & 0.395 & 0 \\
\hline SV1 & 0.273 & 0 & 0.664 & 0 & 0.343 & 0 \\
\hline SV2 & 0 & 0.354 & 0.765 & 0 & 0.366 & 0 \\
\hline RV5 & 0 & 0 & 0 & 0 & 0.741 & 0 \\
\hline RV6 & 0 & 0 & 0.358 & 0 & 0.839 & 0 \\
\hline LA_Area & 0.220 & 0 & 0.591 & 0 & 0 & 0.482 \\
\hline RA_Area & 0 & 0 & 0.893 & 0 & 0 & 0 \\
\hline LVDd & 0.593 & 0 & 0.468 & 0.418 & 0 & 0 \\
\hline IVSd & 0.769 & 0 & 0 & 0 & 0 & 0 \\
\hline LVMass & 0.906 & 0 & 0.228 & 0 & 0 & 0 \\
\hline LVMI & 0.93 & 0 & 0.20 & 0 & 0 & 0 \\
\hline MR & 0 & 0.23 & 0 & 0 & 0 & 0.89 \\
\hline TR & 0 & 0 & 0 & 0 & 0 & 0 \\
\hline MR_VTI & 0 & 0 & 0 & 0 & 0.67 & 0.61 \\
\hline TR_VTI & 0 & 0 & 0 & 0 & 0.52 & 0.30 \\
\hline
\end{tabular}

The factor analysis of the dPCG measurements is far more important, because of the large number of amplitude values of the time-frequency map. The used 170 box values were determined by the parameter reduction of more than 1000 values by the factor analysis.

At the next step the echocardiographic left ventricular end diastolic dimension (LVIDd), the ejection fraction (EF), the left ventricular mass index (LVMI), the aortic Vmax (detecting systolic failure or the grade of aortic stenosis), the rate of mitral and tricuspid regurgitation
(MR, TR) were estimated using SPSS (V15.0) multivariate discriminant analysis (MDA) module.

These output variables converted into three discrete values, the input values were the $170 \mathrm{dPCG}$, and separately, the best dECG parameters determined by the factor analysis. The Wilks' statistic of the MDA model selects the best parameters and the unstandardized canonical discriminant function coefficients were determined (Table 3, 4, and 5 for the dECG input parameters).

Table 3. Canonical discriminant function (CDF) of the unstandardized coefficients for LVIDd estimation.

\begin{tabular}{|c|c|c|}
\hline Parameter & Function 1 & Function 2 \\
\hline QRS T & 0.074438 & -0.02941 \\
\hline R_aVF & -1.21458 & 0.576215 \\
\hline R_V6 & 0.509615 & -0.59927 \\
\hline S V4 & 0.642333 & 2.071545 \\
\hline S V6 & -2.12986 & -1.25901 \\
\hline V1_P-duration & 0.008335 & 0.023761 \\
\hline PIII 2nd T_corr & 0.012306 & -0.00378 \\
\hline PIII 2nd Area & -0.13478 & -0.25712 \\
\hline Constant: & -7.8649 & -0.65885 \\
\hline
\end{tabular}

Table 4. CDF of the unstandardized coefficients for LVMI estimation.

\begin{tabular}{|lrr|}
\hline \multicolumn{1}{|c}{ Parameter } & Function 1 & Function 2 \\
\hline QRS_T & 0.061159 & 0.022874 \\
\hline R_III & -1.60416 & 1.738611 \\
\hline R_aVR & -1.93258 & -1.15135 \\
\hline R_V6 & 0.605695 & -0.92704 \\
\hline PII_2nd_T_corr & -0.01405 & -0.02759 \\
\hline PIII_2nd_T_corr & 0.020277 & 0.01712 \\
\hline Constant: & -5.71712 & -1.48757 \\
\hline
\end{tabular}

Table 5. CDF of the unstandardized coefficients for left atrial area (LA_Area) estimation.

\begin{tabular}{|lrr|}
\hline \multicolumn{1}{|c}{ Parameter } & Function 1 & Function 2 \\
\hline QRS_T & 0.059714 & -0.02554 \\
\hline R_aVR & -2.36806 & -4.0458 \\
\hline S_I & -3.18466 & 1.88948 \\
\hline S_V2 & -1.01791 & 1.842552 \\
\hline S_V3 & 1.994183 & -0.72072 \\
\hline \multicolumn{1}{|c}{ Constant: } & -5.81357 & 1.317417 \\
\hline
\end{tabular}


Table 6. CDF of the unstandardized coefficients for right atrial area (RA_Area) estimation.

\begin{tabular}{|lrr|}
\hline \multicolumn{1}{|c}{ Parameter } & Function 1 & \multicolumn{1}{c|}{ Function 2 } \\
\hline QRS_T & -0.03297 & 0.045872 \\
\hline R_III & 1.172145 & -0.40161 \\
\hline III_P2minmax & -3.32678 & 5.274863 \\
\hline V1_T-to-P1minmax & 0.062558 & -0.00203 \\
\hline V1_P-duration & 0.020982 & 0.020181 \\
\hline PV1_2nd_Tcorr & 0.026194 & -0.0062 \\
\hline \multicolumn{1}{|c}{ Constant: } & -2.68679 & -6.65305 \\
\hline
\end{tabular}

For the LVIDd estimation the model selected 8 variables, the values of the Wilks' lambda decreased from 0.882 to 0.709 ( $p<0.0001$ in each step). For the 3 outputs, the test of function 1 through 2 and 2: Wilks' lambda 0.709 and 0.899 ; chi-square 132.93 and 41.09 (sign. $\mathrm{p}<0.001$ ).

Table 7. Classification results of LVIDd prediction based on dECG data

\begin{tabular}{|lrrrrr|}
\hline & \multicolumn{2}{c}{ Pred. } & & \multicolumn{1}{c|}{ Total } \\
\hline Original & & 1 & 2 & 3 & \\
\hline Count & 1 & 172 & 58 & 36 & 266 \\
\hline & 2 & 29 & 42 & 21 & 92 \\
\hline & 3 & 2 & 8 & 27 & 37 \\
\hline$\%$ & 1 & 64.66 & 21.80 & 13.53 & 100 \\
\hline & 2 & 31.52 & 45.65 & 22.82 & 100 \\
\hline & 3 & 5.405 & 21.62 & 72.97 & 100 \\
\hline
\end{tabular}

The model selected 6 variables for the LVMI estimation, the values of the Wilks' lambda between 0.904 and $0.714(p<0.0001$ in each steps). The test of function 1 through 2 and 2: Wilks' lambda 0.714 and 0.946; chi-square 130.47 and 21.41 (sign. $\mathrm{p}<0.0006$ ).

Table 8. Classification results of LVMI prediction based on dECG data

\begin{tabular}{|lrrrrr|}
\hline & \multicolumn{2}{c}{ Pred. } & \multicolumn{2}{c|}{ Total } \\
\hline Original & & 1 & 2 & 3 & \\
\hline Count & 1 & 55 & 31 & 5 & 91 \\
\hline & 2 & 64 & 126 & 52 & 242 \\
\hline & 3 & 4 & 17 & 41 & 62 \\
\hline$\%$ & 1 & 60.43 & 34.06 & 5.49 & 100 \\
\hline & 2 & 26.44 & 52.06 & 21.48 & 100 \\
\hline & 3 & 6.451 & 27.41 & 66.12 & 100 \\
\hline
\end{tabular}

Five variables were selected for the estimation of LA, and 6 for RA dimensions. The Wilks' lambda for 1 through 2 is 0.798 (chi-square: $87.49, \mathrm{p}<0.001$ ), for 2 is 0.995 (chi-square: $1.752, \mathrm{p}<0.5$ ) for LA, and for RA: $0.731 \quad(121.37, \quad \mathrm{p}<0.001), \quad 0.932 \quad(27.25 \quad \mathrm{p}<0.001)$, respectively.

Table 8. Classification results of left atrial (LA) dimensions with dECG data.

\begin{tabular}{|lrrrrr|}
\hline & \multicolumn{2}{c}{ Pred. } & & \multicolumn{1}{c|}{ Total } \\
\hline Original & & 1 & 2 & 3 & \\
\hline Count & 1 & 194 & 40 & 40 & 274 \\
\hline & 2 & 27 & 40 & 36 & 103 \\
\hline & 3 & 2 & 8 & 8 & 18 \\
\hline$\%$ & 1 & 70.80 & 14.59 & 14.59 & 100 \\
\hline & 2 & 26.21 & 38.83 & 34.95 & 100 \\
\hline & 3 & 11.11 & 44.4 & 44.4 & 100 \\
\hline
\end{tabular}

Table 9. Classification results of right atrial (RA) dimensions based on dECG data.

\begin{tabular}{|lrrrrr|}
\hline & \multicolumn{2}{c}{ Pred. } & & \multicolumn{2}{c|}{ Total } \\
\hline Original & & 1 & 2 & 3 & \\
\hline Count & 1 & 219 & 94 & 11 & 324 \\
\hline & 2 & 18 & 43 & 8 & 69 \\
\hline & 3 & 2 & 2 & 8 & 12 \\
\hline$\%$ & 1 & 67.59 & 29.01 & 3.39 & 100 \\
\hline & 2 & 26.08 & 62.31 & 11.59 & 100 \\
\hline & 3 & 16.66 & 16.66 & 66.66 & 100 \\
\hline
\end{tabular}

Based on the dPCG data (170 values for each cardiac cycle at the apex) the following echocardiographic parameters were estimated: aortic Vmax, ejection fraction (EF), mitral and tricuspid regurgitation (MR, TR). Variables (VAR_) 1-30 represent the S1 (from the highest frequencies to the lowest in 10 steps, in 3 timesegments), 31 to 90 the systole (in 6 time-segments), 91 to 110 the S2 (in 2 time-segments), and 111 to 170 the diastole (in 6 time-segments).

The Vmax parameter is estimated by 17 parameters (test of function 1 through 2: Wilks' lambda 0.111 , chisquare 274.65; function 2: 0.489 , and 89.352 , both $\mathrm{p}<0.001)$. The same parameters for EF are: 7 variables (test of function: 0.391, 121.986, 0.811, 27.23, $\mathrm{p}<0.001$ ); for MR: 7 parameters, (test of function: 0.416, 113.03, $0.78,32.123, \mathrm{p}<0.001$ ); for TR: 7 parameters (test of function: $0.534,81.274,0.903,13.166, \quad \mathrm{p}<0.001$ and $\mathrm{p}<0.02$ ).

Table 10. Classification results of Vmax prediction based on APCG data

\begin{tabular}{|lrrrrr|}
\hline & \multicolumn{2}{c}{ Pred. } & \multicolumn{2}{c|}{ Total } \\
\hline Original & & 1 & 2 & 3 & \\
\hline Count & 1 & 299 & 10 & 2 & 311 \\
\hline & 2 & 16 & 35 & 2 & 53 \\
\hline & 3 & 0 & 1 & 30 & 31 \\
\hline \% & 1 & 96.14 & 3.21 & 0.64 & 100 \\
\hline & 2 & 30.19 & 66.04 & 3.77 & 100 \\
\hline & 3 & 0 & 3.22 & 96.77 & 100 \\
\hline
\end{tabular}


Table 11. LVMI prediction based on APCG data

\begin{tabular}{|lrrrrr|}
\hline & \multicolumn{2}{c}{ Pred. } & & \multicolumn{2}{c|}{ Total } \\
\hline Original & & 1 & 2 & 3 & \\
\hline Count & 1 & 114 & 29 & 18 & 161 \\
\hline & 2 & 21 & 84 & 26 & 131 \\
\hline & 3 & 22 & 27 & 54 & 103 \\
\hline$\%$ & 1 & 70.81 & 18.01 & 11.18 & 100 \\
\hline & 2 & 16.03 & 64.12 & 19.85 & 100 \\
\hline & 3 & 21.36 & 26.21 & 52.43 & 100 \\
\hline
\end{tabular}

Table 12. MDA prediction of mitral regurgitation (MR) with dPCG data

\begin{tabular}{|lrrrrr|}
\hline & \multicolumn{2}{c}{ Pred. } & & \multicolumn{2}{c|}{ Total } \\
\hline Original & & 1 & 2 & 3 & \\
\hline Count & 1 & 191 & 29 & 6 & 226 \\
\hline & 2 & 42 & 69 & 5 & 116 \\
\hline & 3 & 8 & 13 & 32 & 53 \\
\hline$\%$ & 1 & 84.51 & 12.83 & 2.65 & 100 \\
\hline & 2 & 36.21 & 59.48 & 4.31 & 100 \\
\hline & 3 & 15.09 & 24.53 & 60.38 & 100 \\
\hline
\end{tabular}

Table 13. Prediction of tricuspid regurgitation (TR) based on APCG data

\begin{tabular}{|lrrrrr|}
\hline & \multicolumn{2}{c}{ Pred. } & & \multicolumn{2}{c|}{ Total } \\
\hline Original & & 1 & 2 & 3 & \\
\hline Count & 1 & 172 & 50 & 7 & 229 \\
\hline & 2 & 35 & 64 & 6 & 105 \\
\hline & 3 & 4 & 5 & 52 & 61 \\
\hline$\%$ & 1 & 75.11 & 21.83 & 3.05 & 100 \\
\hline & 2 & 33.33 & 60.95 & 5.71 & 100 \\
\hline & 3 & 6.55 & 8.19 & 85.25 & 100 \\
\hline
\end{tabular}

\section{Discussion and conclusions}

Our study showed, that the method of multiple discriminant analysis could adequately predict the most important echocardiographic parameters by the timefrequency measurements of $\mathrm{dPCG}$ and $\mathrm{dECG}$.

Table 14. Canonical discriminant function (CDF) of the unstandardized coefficients for TR estimation with dPCG data.

\begin{tabular}{|lrr|}
\hline Parameter & Function 1 & \multicolumn{1}{c|}{ Function 2 } \\
\hline & 1 & 2 \\
\hline VAR00015 & -1.987 & 1.41 \\
\hline VAR00021 & 0.952 & 0.115 \\
\hline VAR00082 & -0.619 & 0.789 \\
\hline VAR00092 & -0.139 & -0.898 \\
\hline VAR00134 & 3.492 & 0.077 \\
\hline VAR00166 & 1.502 & -0.302 \\
\hline (Constant) & -0.68 & 0.426 \\
\hline
\end{tabular}

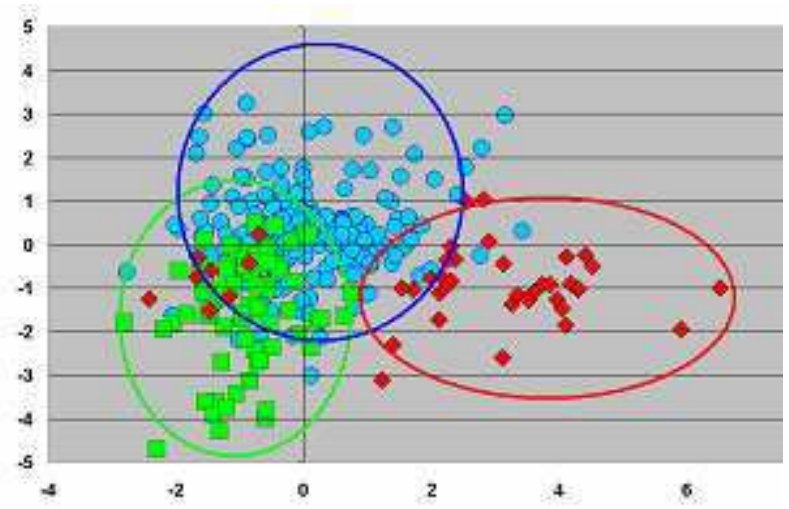

Figure 1. Two-function map for the representation of the discriminant power of the variables and the method of the individual stratification.

Table 14. and Figure 1. show how to use the results of complex statistical method. The two canonical discriminant functions (CDF) are calculated with the new patient's measurements and the unstandardized coefficients. This value will be compared with the use of the circles of the figure. Beside of the prediction (disease severity), the statistical meaning (strength) is also represented by the prediction matrix (in this example see Table 13).

\section{References}

[1] Tavel ME. Cardiac auscultation: a glorious past - and it does have a future! Circulation 2006;113:1255-59.

[2] Kail E. Khoor S. Kail B. Fugedi K. Internet digital phonocardiography in clinical settings and in population screening. Computers in Cardiology 2004;31:501-4.

[3] Kail E. Khoor S. Fugedi K. Kovacs I. et al. Expert system for phonocardiographic monitoring of heart failure patients based on wavelet analysis. Computers in Cardiology 2005;32:833-6.

[4] Khoor S. Kovacs I. Fugedi K. et al. Telemedicine digital phonocardiography: cost-effective strategies in heart failure screening and monitoring. Computers in Cardiology 2007;34:649-652.

[5] Rangayyan RM. Lehner RJ. Phonocardiogram signal analysis: a review. Crit Rev Biomed Eng 1988;15:211-236.

[6] Durand LG. Pibarot P. Digital signal processing of the phonocardiogram: review of the most recent advancements. Crit Rev Biomed Eng 1995;23(3):163-219.

Address for correspondence

Sandor Khoor MD PhD

Szent Istvan Hospital

$3^{\text {rd }}$ Dept K-Int Med

Nagyvarad ter 1

Budapest. Hungary. H-1096

skhoor@gmail.com

sandor.khoor@uva.ca 